\title{
Caracterização do Perfil Cognitivo-Linguístico de Escolares com Dificuldades de Leitura e Escrita
}

\section{Cognitive-Linguistic Profile in Students with Reading and Writing Difficulties}

\author{
Olga Valéria C. A. Andrade*, $a$, Paulo Estêvão Andrade ${ }^{a, b} \&$ Simone Aparecida Capellini ${ }^{b}$ \\ ${ }^{a}$ Colégio Criativo, Marília, São Paulo, Brasil \\ $\&{ }^{b}$ Universidade Estadual Paulista Júlio de Mesquita Filho, Marília, São Paulo, Brasil
}

\begin{abstract}
Resumo
Investigou-se o perfil cognitivo-linguístico de escolares com dificuldades na aquisição da linguagem escrita acrescentando-se testes específicos em um modelo de resposta à intervenção (identificação e intervenção precoces do risco para transtorno de leitura). Participaram 45 escolares do $2^{\circ}$ ano, idade média de 7 anos de ambos os gêneros, divididos em grupo controle (sem dificuldades, $n=32$ ) e grupo de risco (com dificuldades, $n=13$ ). Escores mais de 1,65 DP abaixo da média do grupo controle para cada teste foram considerados déficits específicos. Enquanto a análise de grupo apontou os prejuízos fonológicos como único fator de risco, a individual acrescentou a inatenção e a nomeação rápida como fatores causais independentes. Um modelo ampliado de resposta à intervenção que inclui a avaliação cognitiva é proposto.

Palavras-chave: Avaliação, aprendizagem, cognição, leitura, escrita.
\end{abstract}

\begin{abstract}
The cognitive-linguistic profile of students with reading difficulties was investigated by adding specific tests to a response to intervention model (early identification and intervention for reading disorders). Forty-five second graders of both sexes and mean age of 7 years were divided into control group (without reading difficulties, $n=32$ ) and at-risk group (with reading difficulties, $n=13$ ). Scores below the mean of the control group $(S D>1.65)$ for each test were considered as specific deficits. Whereas group analysis showed phonological impairments as the only risk factor, an individual analysis added inattention and rapid naming as independent causal factors. An extended response model to the intervention, which includes cognitive assessment, is proposed.

Keywords: Assessment, learning, cognition, reading, writing.
\end{abstract}

A literatura recente indica que $36 \%$ das crianças do quarto ano nos Estados Unidos estão lendo abaixo dos níveis básicos (Shaywitz \& Shaywitz, 2008). Na literatura nacional reporta-se que cerca de $30 \%$ dos escolares nas primeiras séries do ensino fundamental de escolas públicas e particulares apresentam algum tipo de dificuldade de aprendizagem (Alves \& Ribeiro, 2011; Bicalho \& Alves, 2010; Refundini, Martins, \& Capellini, 2010).

De um modo geral, o baixo rendimento escolar pode ser decorrente de fatores extrínsecos ou ambientais, tais como desvantagens sociais-econômicas-culturais, pedagógicas e/ou socioafetivas e é referido como "dificuldade de aprendizagem", ou então ser decorrente de fatores intrínsecos ao escolar, como disfunções cognitivas de origem genético-neurológica referidas como "distúrbio" ou "transtorno de aprendizagem" (TA; Alves, Mousinho, \& Capellini, 2011; Ciasca, 2004).

\footnotetext{
"Endereço para correspondência: Colégio Criativo, Unidade Tangará, Rua das Safiras, 45, Marília, SP, Brasil 17516-130. E-mail: olguinhavandrade@yahoo.com, paulo_sustain@yahoo.com e sacap@uol.com.br
}

Os TA são identificados por um baixo rendimento inesperado em testes padronizados e individualmente administrados de leitura, denominado de transtorno de leitura (TL) ou dislexia, de matemática (transtorno da matemática ou discalculia) ou expressão escrita (comprometimento da ortografia e caligrafia, mas geralmente associada a outros transtornos), bem como o transtorno da aprendizagem sem outra especificação, ou distúrbio de aprendizagem (prejuízos cognitivos mais gerais de processamento e armazenamento da informação que afetam a linguagem oral, a linguagem escrita e a matemática). A noção de inesperado é central aos transtornos de aprendizagem, pois estes ocorrem apesar de inteligência normal, adequadas oportunidades educacionais, socioeconômicas e afetivas, bem como na ausência de transtornos sensoriais e neurológicos aparentes. Se considerarmos o baixo rendimento escolar associado aos transtornos de aprendizagem, o percentual cai de $30 \%$ para uma média de 2 a $10 \%$ da população estudantil, dependendo do tipo de TA (American Psychiatric Association [APA], 2002).

Entretanto, enquanto um diagnóstico definitivo não for realizado não é possível determinar se o baixo ren- 
Andrade, O. V. C. A., Andrade, P. E. \& Capellini, S. A. (2014). Caracterização do Perfil Cognitivo-Linguístico de Escolares com Dificuldades de Leitura e Escrita.

dimento trata-se de uma dificuldade de aprendizagem ou um transtorno. Neste artigo os casos pré-diagnósticos de baixo rendimento na aquisição e desenvolvimento da linguagem escrita serão referenciados simplesmente como dificuldades de leitura sendo que as dificuldades de escrita estão subentendidas.

O presente estudo será focado nas dificuldades de leitura porque o prejuízo significativo na aquisição da linguagem escrita não é exclusivo da dislexia, mas sim um sintoma pervasivo que está presente em mais de $80 \%$ dos diferentes tipos de Transtorno de Aprendizagem (Fuchs \& Fuchs, 2006; Shaywitz \& Shaywitz, 2008). Devido a essa presença pervasiva de prejuízos de leitura nos transtornos de aprendizagem, bem como no TDAH, os escolares com dificuldades (ou baixo rendimento) de leitura serão considerados de risco para Transtorno de Aprendizagem (APA, 2002).

Por outro lado, o Transtorno do Déficit de Atenção/ Hiperatividade (TDA/H), o transtorno neuropsiquiátrico mais comum na infância), embora não seja considerado um TA, geralmente está associado a dificuldades na aquisição e desenvolvimento da linguagem escrita, particularmente nos subtipos com predomínio da desatenção (TDA) e combinado (TDAH) (Siqueira et al., 2011). Hoje se reconhece que a desatenção (ou inatenção) está frequentemente associada a prejuízos de nomeação automática rápida (RAN) e memória de trabalho fonológica (MTF) que são sintomas típicos da dislexia, além de prejuízos de consciência fonológica (Martinussen \& Tannock, 2006; Willcutt et al., 2010). De fato, 20 a $40 \%$ dos indivíduos TDAH também apresentam prejuízos na linguagem escrita (Willcutt et al., 2010; Willcutt, Pennington, Olson, \& DeFries, 2007).

Todos concordam que a identificação e prevenção precoces de escolares de risco para transtornos de aprendizagem é um procedimento pré-diagnóstico fundamental, pois permite aproveitar uma fase de alta plasticidade dos sistemas neurocognitivos envolvidos na aquisição da linguagem escrita ao mesmo tempo em que se evita o desenvolvimento de uma lacuna escolar demasiada (Alves, Siqueira, Lodi, \& Araújo, 2011; Catts, Fey, Zhang, \& Tomblin, 2001; Fletcher \& Vaughn, 2009; Reynolds \& Shaywitz, 2009).

No entanto, toda a abordagem clínica atual é baseada na noção do "inesperado", que é central ao conceito de transtornos de aprendizagem (Fletcher \& Lyon, 2008; Reynolds \& Shaywitz, 2009) e da qual originou-se a abordagem diagnóstica baseada na discrepância QI-rendimento. Esta abordagem requer que o rendimento em um dado domínio acadêmico (com base em testes referenciados em normas) esteja a um ou dois desvios-padrão abaixo da média do QI geral para que o escolar seja diagnosticado e encaminhado para programas de intervenção (Shaywitz, Morris, \& Shaywitz, 2008).

O grande problema da abordagem de discrepância QI-rendimento é que o diagnóstico definitivo é possível somente por volta do $4^{\circ}$ ano, quando as eventuais discrepâncias entre desempenho acadêmico e QI podem ser confirmadas com segurança. Isto torna este modelo clínico inviável, não porque a noção de discrepância esteja errada, "mas sim com o efeito prático na vida real de se implementar este modelo em um ambiente de escola primária", pois a espera pela discrepância caracteriza uma "espera pelo fracasso" (Shaywitz et al., 2008, p. 454). Em outras palavras, a espera pela discrepância QI-rendimento e pelo diagnóstico definitivo no final da primeira metade ou no início da segunda metade do ensino fundamental não prioriza a identificação e intervenção precoce do escolar de risco, privando-o de receber as intervenções preventivas e remediativas adequadas à suas dificuldades em um período de maior plasticidade neurocognitiva, gerando-se uma lacuna escolar (defasagem em relação ao seu grupo-classe) maior e mais difícil de ser superada (Andrade, Prado, \& Capellini, 2011; Fuchs \& Fuchs, 2006).

Uma alternativa para o modelo de discrepância, a qual vem recebendo enorme atenção nos últimos anos, são os modelos de "resposta à intervenção", ou RTI, caracterizados pela identificação e intervenção precoces (Fletcher \& Vaughn, 2009; Fuchs \& Fuchs, 2006). Inicialmente, procede-se ao rastreamento precoce de escolares de risco por meio de avaliações baseadas no currículo (linguagem escrita e matemática), principalmente a leitura, considera-se, de um modo geral, como baixo rendimento (identificação do risco) os desempenhos que ficam no percentil 25 ou 1 DP abaixo da média (grupo-classe ou testes normatizados; Fuchs \& Fuchs, 2006). As intervenções são normalmente hierarquizadas em três "camadas".

A primeira camada da abordagem RTI caracteriza-se pela intervenção coletiva por meio de estratégias pedagógicas de qualidade (baseadas em evidência) na sala de aula (intervenção primária). Na segunda camada as intervenções são realizadas em grupos de 3 a 4 alunos (intervenção secundária). Na terceira camada a intervenção é feita com grupos menores ou atendimento individualizado (intervenção terciária). A passagem de uma camada a outra depende da "responsividade" adequada ou inadequada após cada camada, e a persistência da baixa responsividade até a terceira camada é considerada TA (Fletcher \& Vaughn, 2009; Fuchs \& Fuchs, 2006).

No entanto, os principais modelos RTI (Fletcher \& Vaughn, 2009; Fuchs \& Fuchs, 2006) defendem que o rastreamento deve ser feito principalmente por avaliações baseadas no currículo (principalmente a leitura) e não pelo baixo rendimento ou diferenças intraindividuais em testes padronizados (normativos ou baseados em critério). Argumenta-se que estas avaliações não permitem distinguir o baixo rendimento resultante de um real TA ou de outros problemas, como socioeconômicos, afetivos ou instrucionais (Fletcher \& Lyon, 2008). Argumenta-se também que a determinação do perfil cognitivo de forças e fraquezas no modelo intraindividual em nada acrescenta ao rastreamento de risco para TA e ao planejamento da intervenção: uma vez que os problemas de leitura e escrita na sua maioria estão associados a problemas fonológicos o rastreamento por problemas fonológicos em nada acrescenta, bastando 
fazer o rastreamento somente com base na leitura, o que reduziria o tempo e o custo gastos na identificação de escolares de risco (Fletcher \& Lyon, 2008).

O modelo de RTI tem sido duramente criticado por assumir que o rastreamento deve ser feito somente com medidas baseadas no currículo (primordialmente a leitura) e que não há necessidade de uma caracterização dos prejuízos cognitivo-linguísticos dos escolares de risco com dificuldades de aprendizagem (Reynolds \& Shaywitz, 2009). Entretanto, a literatura está repleta de evidências mostrando que o perfil de forças e fraquezas psicológicas e cognitivas no TA se reflete em padrões neuroanatômico-funcionais igualmente típicos (Reynolds \& Shaywitz, 2009), e que os prejuízos de leitura podem ter diferentes causas, como déficits fonológicos ou atencionais, etc. (Willcutt et al., 2010). Por isso, a identificação do perfil cognitivo-linguístico é fundamental na elaboração de estratégias pedagógicas diferenciais, adequadas às necessidades específicas de cada aluno (Reynolds \& Shaywitz, 2009). Sabemos por experiência que o manejo comportamental diferencial, por exemplo, para casos de baixo rendimento associado à desatenção e/ou hiperatividade, é fundamental.

O presente estudo acrescenta a caracterização do perfil cognitivo-linguístico dos escolares com dificuldades e sem dificuldades de leitura ainda no início do $2^{\circ}$ ano em um modelo de resposta à intervenção. Portanto, este estudo tem por objetivo ampliar as possibilidades de transformação deste modelo para uma abordagem ampliada em que a avaliação do perfil cognitivo-linguístico permita a identificação de forças e fraquezas dos escolares de risco $\mathrm{e}$, consequentemente, possibilite o desenvolvimento de programas de intervenção que atendam mais efetivamente suas necessidades e potencialidades individuais.

\section{Método}

\section{Participantes}

Participaram deste estudo de delineamento caso-controle longitudinal 45 escolares que na fase inicial estavam no início do $2^{\circ}$ ano do ensino fundamental, com idade média de 7 anos e 4 meses, sendo 29 do gênero masculino, matriculados em quatro salas de aula de uma escola da rede particular de ensino da cidade de Marília - SP. Os participantes são provenientes da classe média-alta, cujo status socioeconômico corresponde às classes $\mathrm{A} \mathrm{e} \mathrm{B}$ de acordo com a Escala para Avaliação do Status Sócio-Econômico da Associação Brasileira dos Institutos de Pesquisa de Mercado - ABIPEME (Almeida \& Wickerhauser, 1991). Optamos por um grupo de escolares de classe média-alta objetivando minimizar o máximo possível o peso dos aspectos socioeconômicos, culturais (Demo, 2007) e pedagógicos (Capellini et al., 2009) desfavoráveis, fatores que contribuem para maior ocorrência de problemas de aprendizagem na rede pública, cujo desempenho acadêmico é notadamente inferior ao da particular (Alves \& Ribeiro, 2011; Bicalho \& Alves, 2010; Demo, 2007).
Os critérios para inclusão foram (a) a assinatura pelos pais ou responsáveis do Termo de Consentimento Livre e Esclarecido, (b) os escolares apresentarem acuidade visual, auditiva e desempenho cognitivo dentro dos padrões de normalidade e nenhum histórico de danos neurológicos descrito no prontuário escolar. Os critérios de exclusão foram (a) a ausência da assinatura do Termo Livre e Esclarecido, (b) suspeita de déficits sensoriais (auditivo e/ou visual) não corrigidos, (c) histórico de problemas neurológicos adquiridos, (d) portadores de necessidades educativas especiais, em regime de inclusão.

\section{Material}

Avaliação Diagnóstica (AD). Todos os escolares do presente estudo participaram da AD. Na escola em que se realizou este estudo a $\mathrm{AD}$ inclui atividades pedagógicas especialmente desenvolvidas para avaliar o desempenho em domínios acadêmicos básicos, incluindo habilidades de linguagem escrita, matemática, ciência, geografia e história (Luckesi, 2005).

Para o propósito específico da presente investigação e consistentemente com as abordagens RTI (Fletcher \& Vaughn, 2009; Fuchs \& Fuchs, 2006) focamos unicamente as avaliações das habilidades de leitura e escrita da $\mathrm{AD}$, descritas a seguir: (a) Leitura: fluência e acurácia na leitura em voz alta de palavras simples monossílabas, dissílabas e trissílabas extraídas dos livros didáticos e apostilas usadas pela escola; (b) Leitura silenciosa: desenhar o significado de uma palavra escrita; (c) Escrita: escrever todas as letras do alfabeto que conhece (letra de forma ou cursiva), fazer um círculo vermelho em volta das vogais e um círculo azul das consoantes, escrever com qual letra começa o nome de uma figura apresentada; (d) Conhecimento de sílabas: escrever a sílaba correta para completar uma palavra; (e) Conhecimento de palavras: formar, usando o alfabeto móvel, o maior número possível de palavras que conhece e depois escrevê-las no papel; (f) Escrita: escrever palavras ditadas pelo examinador; (g) compreensão da linguagem oral: ao acabar de ouvir uma história, desenhar o que compreendeu;

Teste de Desempenho Cognitivo-Linguístico (TDCL; Capellini \& Smythe, 2008).

1. Versão coletiva. Foram usados 3 subtestes: (a) Conhecimento do alfabeto (ALF): letras do alfabeto que o escolar conhece e pode escrever; escore máximo: 26); (b) Cópia de formas (COP): copiar um círculo, quadrado e losango e uma forma complexa combinando elementos destas figuras simples; escore máximo de acordo com escala padronizada: 7; (c) Escrita sob ditado (ESC): 30 palavras reais e 10 pseudopalavras ditadas pelo examinador; escore máximo: 40.

2. Versão individual. Consiste em cinco testes que avaliam: (a) Leitura (LEIT): leitura de palavras e pseudopalavras: fluência (palavras corretamente lidas em um minuto) e acurácia (palavras corretamente lidas de uma lista de 70 palavras; (b) Consciência 
Andrade, O. V. C. A., Andrade, P. E. \& Capellini, S. A. (2014). Caracterização do Perfil Cognitivo-Linguístico de Escolares com Dificuldades de Leitura e Escrita.

fonológica (CFO): identificação de rima (de três palavras ouvidas dizer quais as duas que rimam), aliteração (de três palavras ouvidas dizer quais as que começam com o mesmo som) e segmentação silábica (bater uma palma para cada sílaba de uma palavra ouvida); (c) Processamento auditivo: memória de trabalho fonológica (MTF; repetição de 7 sequências contendo de 2 a 5 palavras), repetição de pseudopalavras (RPs) e discriminação auditiva ou fonêmica, dizer se duas palavras ouvidas, eventualmente diferentes em apenas um fonema, são iguais ou diferentes (DF); (d) Processamento visual: inclui a memória de trabalho visual para formas (MV; reproduzir, na mesma ordem mostrada pelo examinador, sequências de 2 a 5 figuras com detalhes) e memória de trabalho visuoespacial (MVE; número de erros de rotação cometidos na tarefa de memória visual); (e) Velocidade de processamento: nomeação rápida de figuras e dígitos (RAN). Para maiores detalhes veja Capellini e Smythe (2008). Nas Tabelas 2 e 3 essas provas serão referidas somente pelas respectivas siglas.

A versão coletiva do TDCL foi aplicada durante as aulas, com duração de aproximadamente 50 minutos. Os alunos receberam explicações e treino, garantindo o entendimento em cada subteste, bem como instruções orais relativas a aspectos de organização, tais como: não se levantarem do lugar durante o teste, não perguntarem durante a realização da prova e seguirem em frente, mesmo diante de dúvidas ou erros. Na versão individual do TDCL, cada aluno foi recebido em uma sala bem iluminada com o mínimo de ruído possível, considerando o ambiente escolar, com mesa e cadeiras para o aplicador e o aluno; cada sessão teve duração de aproximadamente 40 a 60 minutos. Todas as provas foram precedidas de explicação e treino, conforme orientado no TDCL (Capellini \& Smythe, 2008).

\section{Procedimentos}

Foram entregues 69 termos de consentimento livre e esclarecido a todos os alunos matriculados em quatros salas do $2^{\circ}$ ano do ensino fundamental, dos quais $24,6 \%$ (17 escolares) apresentavam queixas de dificuldades de aprendizagem de acordo com o prontuário escolar que incluiu informações dos pais ou responsáveis e dos professores, assim como a avaliação pedagógica diagnóstica. Dos 69 termos de consentimento entregues 45 retornaram assinados.

Grupos de Risco e Sem Risco para Transtorno de Leitura. Após o recebimento dos termos de consentimento livre e esclarecido foi realizada a análise do prontuário escolar e da Avaliação Diagnóstica (AD) de rotina realizada nesta escola todo primeiro bimestre do ano letivo, quando os sujeitos estavam iniciando o $2^{\circ}$ ano do fundamental $\left(\mathrm{AD}^{\mathrm{I}}\right)$. A partir deste primeiro procedimento com a avaliação diagnóstica, dividimos a amostra em dois grupos: (a) o grupo de risco para transtorno de leitura (GR), composto por 13 escolares identificados pela avaliação diagnóstica como de baixo rendimento, e (b) o grupo sem risco ou controle (GC) composto por 32 escolares. No ano seguinte, no início do $3^{\circ}$ ano os participantes também se submeteram à avaliação diagnóstica de rotina $\left(\mathrm{AD}^{\mathrm{II}}\right)$.

Teste de Desempenho Cognitivo-Linguístico. Foi realizado o Teste de Desempenho Cognitivo-Linguístico (TDCL) nas versões, coletiva e individual (Capellini \& Smythe, 2008) em ambos os grupos, no final do primeiro bimestre do ano letivo em que se iniciou o estudo (TDCL ${ }^{\mathrm{I}}$ ) e um ano depois (TDCL ${ }^{\text {II }}$ ), quando o mesmo grupo de escolares estava cursando o primeiro bimestre do $3^{\circ}$ ano do ensino fundamental. No TDCL ${ }^{\mathrm{II}}$, porém, somente as provas de leitura (LEIT ${ }^{\mathrm{II}}$ ), consciência fonológica (CFO$\mathrm{N}^{\mathrm{II}}$ ), memória de trabalho fonológica $\left(\mathrm{MTF}^{\mathrm{II}}\right)$ e nomeação rápida ( $\mathrm{RAN}^{\mathrm{II}}$ ), por estas terem apresentado as correlações mais fortes e terem sido os principais preditores do baixo desempenho no ano seguinte. No TDCL ${ }^{\mathrm{II}}$ dois alunos não participaram dos testes $(n=30)$ e dois alunos do GR saíram do grupo de risco $(n=11)$, totalizando 41 participantes.

Análise Individual do Perfil Cognitivo-Linguístico (PCL). Optamos por um rigoroso critério de desvio sugerido por Ramus et al. (2003), segundo o qual considera-se como baixo rendimento os escores mais de $1,65 \mathrm{DP}$ abaixo da média de um grupo controle para uma dada tarefa. Portanto, primeiramente obtivemos grupos controle para cada uma das medidas utilizadas do TDCL separando os participantes com escores acima do limite inferior do intervalo de confiança (LIC) de $95 \%$, aos quais chamamos de grupos controle específicos de tarefas (GCE).

Assim, com base nos GCE obtivemos os grupos de risco para as habilidades de leitura e escrita, e também os escolares com prejuízos em cada uma das provas do TDCL selecionando os escores com ou mais de 1,65 DP abaixo da média obtida de cada GCE. De acordo com Ramus e colaboradores em uma distribuição normal os escores 1,65 $D P$ abaixo da média representam desempenhos iguais ou abaixo do quinto percentil $\left(P_{5}\right)$, um limite razoável para se determinar o desvio da média (Ramus et al., 2003, p. 851). O procedimento de se obter grupos controle específicos de tarefa permite-nos identificar os escores desviantes em cada domínio cognitivo e, consequentemente, verificar as diferenças individuais e o perfil de forças e fraquezas dos alunos de risco, dados essenciais do ponto de vista da intervenção (Reynolds \& Shaywitz, 2009).

Esses procedimentos permitiram, em primeiro lugar, acompanhar o desenvolvimento no período de um ano dos escolares de ambos os grupos, os possíveis efeitos da intervenção psicopedagógica realizada pela escola e, finalmente, verificar quais alunos saíram e quais continuaram com risco para TL. Em segundo lugar, permitiram uma caracterização do perfil individual de cada escolar de risco para TL.

Abordagem de Resposta à Intervenção (RTI). Os casos de escolares com base no baixo desempenho na AD, após serem devidamente discutidos e analisados pelo professor e pela coordenação pedagógica, foram indicados para as aulas de apoio. Este procedimento, adotado sistematica- 
mente pela escola na qual se realizou este estudo, é similar aos modelos de "resposta à intervenção" (RI; Fletcher \& Vaughn, 2009; Fuchs \& Fuchs, 2006).

\section{Análise de Dados}

Todas as análises estatísticas foram realizadas com o programa Package for Social Sciences (SPSS) na versão 16.0.

Análise Descritiva. Foram obtidos a média, o desvio padrão e o limite inferior do intervalo de confiança do grupo total e dos grupos de risco específicos de tarefa ou experimentais (GCEs).

Análise Correlacional. Por meio do teste de Kolmogorov-Smirnov e dos requisitos de normalidade com base nos coeficientes de curtose e assimetria sugeridos por Foster (1986) constatou-se que das 15 variáveis investigadas apenas uma não poderia ser assumida como possuindo uma distribuição normal. Portanto, realizamos as análises de correlação entre as provas do TDCL controlando pela idade (Pearson), adotando o nível de significância igual ou menor que $5 \%(0,05)$. Consideramos principalmente as correlações moderadas $(0,40$ a $0,59)$ e moderadas a altas $(r \geq 0,60)$ porque as correlações fracas a moderadas $(0,30$ a 0,39$)$ podem perder a significância com a correção de Bonferroni para falsos positivos (Ramus et al., 2003).

Comparação Intergrupos e Intragrupo. Com base nos testes de normalidade acima citados, o teste $t$ de Student foi utilizado para comparação das médias entre os dois grupos (com e sem dificuldades de leitura), controlado pela igualdade de variâncias com o teste de Levene. Para comparação das médias do mesmo grupo no início e no final do estudo (um ano depois) utilizou-se o teste não-paramétrico de Wilcoxon (teste de igualdade de duas médias para amostras emparelhadas).

Análise Individual. Foram adotados os seguintes critérios de desvio da normalidade: os grupos controle específicos de tarefa (GCE), representados pela média dos escores acima do limite inferior do intervalo de confiança (IC) em cada tarefa, e os escores mais de 1,65 DP abaixo da média do GCE (que representam os grupos de baixo rendimento nas tarefas do TDCL).

\section{Resultados}

O conhecimento do alfabeto avaliado no TDCL ${ }^{\mathrm{I}}$ correlacionou-se somente com a leitura e escrita $(r=0,52, p<$ $0,001)$ e com a memória verbal de trabalho $(r=0,46, p<$ $0,003 ; r=0,35, p<0,024)$, mas não apresentou nenhuma correlação significante no TDCL ${ }^{\mathrm{II}}$.

Três tarefas de processamento fonológico do $\mathrm{TDCL}^{\mathrm{I}}$ tiveram correlações moderadas a altas com o escore composto das habilidades de leitura e escrita no TDCL ${ }^{\mathrm{I}}$ e com as habilidades de leitura (as provas de escrita não foram realizadas por problemas de falta de horário na escola) no TDCL ${ }^{\mathrm{II}}$, a saber: a consciência fonológica (soma dos escores de segmentação silábica, aliteração e rima; $r=0,63 ; p$ $<0,001 ; r=0,58 ; p=0,001$ ), a nomeação rápida (figuras e dígitos; $r=-0,75, p<0,001 ; r=0,70, p<0,001)$ e a memória de trabalho fonológica $(r=0,51, p=0,001 ; r=$ $0,55, p=0,001)$. Enquanto a tarefa de segmentação silábica não se correlacionou com as habilidades de leitura-escrita em nenhuma das duas medições do TDCL, o escore combinado das tarefas de ataque e rima produziu correlações mais altas com as habilidades de leitura e escrita no TDCL ${ }^{\mathrm{I}}$ $(r=0,65, \mathrm{p}<0,001)$ e com as de leitura no TDCL ${ }^{\mathrm{II}}(r=$ $0,57, \mathrm{p}<0,001)$ do que a consciência fonológica como um todo (incluindo a segmentação silábica).

As tarefas de processamento visual não apresentaram nenhuma correlação relevante com nenhuma das tarefas de leitura e escrita nem com as tarefas de processamento fonológico, exceto a memória de trabalho visual que se correlacionou com a memória fonológica de trabalho $(r=$ $0,43, p<0,007)$ somente no TDCLI.

A comparação dos desempenhos do grupo controle (GC) e de risco (GR) realizada com o teste $t$ (Tabela 1) não encontrou efeito de grupo no conhecimento do alfabeto no TDCL ${ }^{\mathrm{I}}$, embora $53,8 \%$ dos escolares do GR pontuaram abaixo do ponto de corte (Tabela 2). Como esperado o teste $t$ apontou um desempenho significantemente superior do GC nas habilidades de leitura e escrita e de consciência fonológica no $\mathrm{TDCL}^{\mathrm{I}}$ (início do estudo no $2^{\circ}$ ano) e no TDCL ${ }^{\text {II }}$ (um ano depois no início do $3^{\circ}$ ano), exceto a segmentação silábica que no TDCL ${ }^{\mathrm{II}}$ tornou-se estatisticamente igual para ambos os grupos. Na Tabela 2 todos os escolares do GR apresentaram escores abaixo do ponto de corte nas tarefas de leitura, $12(92,3 \%)$ nas de escrita, e $10(76,9 \%)$ nas tarefas de consciência fonológica, o que demonstra uma alta sensitividade destas provas do TDCL aos escolares de risco.

O desempenho na nomeação, memória de trabalho visual e memória de trabalho fonológica também foram superiores no $\mathrm{GC}$ em ambas as medições, $\mathrm{TDCL}^{\mathrm{I}} \mathrm{e} \mathrm{TDCL}^{\mathrm{II}}$ (Tabela 1). O teste $t$ não diferenciou os grupos GC e GR na cópia de formas e memória indireta de dígitos, apesar de os respectivos escores abaixo do ponto de corte corresponderem a $61,5 \%$ e $46,1 \%$ do GR, respectivamente (Tabela 1). Também não houve efeito de grupo para as tarefas de repetição de pseudopalavras e discriminação auditiva, cópia de formas e memória de trabalho visuoespacial (Tabela 1).

Para confirmar os efeitos de grupo obtidos por meio do teste $t$ utilizamos o teste de Kruskal-Wallis, uma análise de variância (ANOVA) não-paramétrica com postos sinalizados (como no teste de Wilcoxon) usada para duas ou mais amostras independentes, o qual permite controlar o efeito das múltiplas comparações. O teste de Kruskal-Wallis corroborou as diferenças entre os grupos para as habilidades de leitura e escrita no $\operatorname{TDCL}^{\mathrm{I}}[\chi 2(1, N=45)=$ $26,88 ; p<0,001]$ e de leitura no TDCL ${ }^{\mathrm{II}}\left[\chi^{2}(1, N=41)=\right.$ $22,9 ; p<0,001]$, bem como em ambas as medições (TDCL $^{\mathrm{I}}$ e TDCL ${ }^{\text {II) }}$ para as habilidades de consciência fonológica $[\chi 2(1, N=45)=19,57 ; p<0,001 ; \mathrm{e} \div 2(1, N=41)=18,51$; $p<0,001]$, memória fonológica de trabalho $\left[\chi^{2}(1, N=\right.$ $45)=13,29 ; p<0,001 ; \mathrm{e} \div 2(1, N=41)=4,14 ; p<0,42]$ e nomeação $[\chi 2(1, N=45)=8,02 ; p<0,005 ; \mathrm{e} \div 2(1, N$ $=41)=4,03 ; p<0,45]$. 
Andrade, O. V. C. A., Andrade, P. E. \& Capellini, S. A. (2014). Caracterização do Perfil Cognitivo-Linguístico de Escolares com Dificuldades de Leitura e Escrita.

Tabela 1

Média e DP dos Grupos GC e GR no TDCLI e TDCLII e as Diferenças Significativas (*)

\begin{tabular}{|c|c|c|c|c|}
\hline Testes & $\mathrm{GC}(n=32)$ & $\operatorname{GR}(n=13)$ & Teste $t$ & $p<0,05$ \\
\hline Alfabeto & $25,09(1,90)$ & $22,92(3,77)$ & 1,974 & 0,68 \\
\hline Leitura & $119,00(13,03)$ & $70,85(28,15)$ & 5,915 & $0,000^{*}$ \\
\hline Leitura ${ }^{\mathrm{II}}$ & $136,9(19,09)$ & $100,17(12,38)$ & 6,068 & $0,000^{*}$ \\
\hline Escrita & $34,12(3,26)$ & $20,15(8,78)$ & 5,581 & $0.000 *$ \\
\hline Leitura/Escrita & $153,41(14,78)$ & $91,00(35,54)$ & 6,092 & $0,000^{*}$ \\
\hline Consciência Fonológica & $38,22(2,79)$ & $31,38(4,21)$ & 6,391 & $0,000^{*}$ \\
\hline Segmentação Silábica & $11,72(0,63)$ & $11.08(1,15)$ & 2,445 & $0,019 *$ \\
\hline Ataque/Rima & $26,5(2.56)$ & $20,31(3.66)$ & 6,464 & $0,000^{*}$ \\
\hline Consciência Fonológica II & $39,48(1,86)$ & $32,00(6,68)$ & 3,815 & $0,003 *$ \\
\hline Segmentação Silábica ${ }^{\mathrm{II}}$ & $11,86(0.44)$ & $11,00(1,59)$ & 11,702 & 0,091 \\
\hline Ataque/Rima ${ }^{\mathrm{II}}$ & $27,62(1.82)$ & $21,00(6,06)$ & 3,716 & $0,003 *$ \\
\hline Memória de Trabalho Fonológica & $4,16(1,01)$ & $2,77(0,83)$ & 4,344 & $0,000 *$ \\
\hline Mem. de Trabalho FonológicaII & $4,62(1,08)$ & $3,75(1,05)$ & 2,359 & $0,023 *$ \\
\hline Mem. Indireta de Dígitos & $4,75(1,437)$ & $3,85(1,625)$ & 1,842 & 0,072 \\
\hline Repetição de Pseudopalavras & $21,03(2,250)$ & $20,08(1,553)$ & 1,396 & 0,170 \\
\hline Discriminação auditiva & $18,88(0,33)$ & $18,00(1,63)$ & 1,916 & 0,079 \\
\hline Cópia de Formas & $5,19(1,83)$ & $4,00(2,61)$ & 1,732 & 0,090 \\
\hline Mem. de Trabalho Visual & $5,84(1,13)$ & $5,08(0,95)$ & 2,138 & $0,038^{*}$ \\
\hline Mem. de Trab. Visuoespacial & $2,12(3,07)$ & $3,08(3,27)$ & $-0,923$ & 0,361 \\
\hline Nomeação & $78,44(11,73)$ & $97,62(23,89)$ & 2,762 & $0,015^{*}$ \\
\hline NomeaçãoII & $69,90(11,98)$ & $81.25(14,44)$ & $-2,599$ & $0,013 *$ \\
\hline
\end{tabular}

Uma análise minuciosa da Tabela 2 permite-nos dividir o GR em grupos distintos com base no perfil cognitivo-linguístico de cada escolar. Inicialmente podemos destacar dois grupos majoritários, um grupo de 6 escolares de risco $(46,1 \%)$ com baixo rendimento somente em tarefas de processamento fonológico e outro grupo de 6 alunos $(46,1 \%)$ com prejuízos em ambos os processamentos fonológico e visual, dos quais 5 com prejuízos na memória de trabalho visuoespacial, a principal evidência da inatenção no TDA/H (Castellanos, Sonuga-Barke, Milham, \& Tannock, 2006). De fato, o prejuízo na MVE desses escolares coincide com suas características de TDA/H reportadas pelo prontuário escolar e baseadas na escala de Benczik (Benczik, 2000), resultados consistentes com a alta incidência de comorbidade TL-TDA/H reportada na literatura (Siqueira et al., 2011; Willcutt et al., 2010; Willcutt et al., 2007).

Portanto, o déficit na consciência fonológica foi o sintoma prevalente nos alunos do GR, seguido por prejuízos na COP e na RAN. Os prejuízos na MID, MVE, MTF e $\mathrm{MV}$, estiveram presentes em menos de $50 \%$ do GR. Apenas dois dos escolares com prejuízos no processamento fonológico não estavam aparentemente prejudicados na CFO, mas apresentaram prejuízos na MTF e/ou na RAN, em um deles associados a prejuízos na MVE.

As diferenças intragrupo foram obtidas pelo teste de postos sinalizados de Wilcoxon $(p<0,05)$ nos dois momentos do estudo, TDCL ${ }^{\mathrm{I}}$ e TDCL ${ }^{\mathrm{II}}$. Após um ano houve melhoras muito significativas nas habilidades de leitura $\mathrm{e}$ de RAN, respectivamente, em ambos os grupos $\mathrm{GC}(p<$ 
Tabela 2

Escolares do Grupo de Risco (GR), seus Escores no TDCL $L^{I}$ Abaixo do Ponto de Corte Indicadas por $^{a}$, e as Provas do $T_{D C L}{ }^{I I}$ Abaixo do Ponto de Corte ${ }^{\text {II }}$ Indicadas por ${ }^{b}$

\begin{tabular}{|c|c|c|c|c|c|c|c|c|c|c|c|}
\hline & ALF & LEIT & ESC & LE & FON & MTF & MID & COP & MV & MVE & RAN \\
\hline$M$-GCE & 25,56 & 120 & 34,15 & 151,41 & 38,65 & 4 & 5,12 & 5,91 & 5,95 & 1,28 & 75,79 \\
\hline$D P-G C E$ & 0,652 & 12,03 & 3,07 & 15,93 & 2,28 & 0,96 & 1,17 & 1,28 & 0,89 & 0,89 & 8,79 \\
\hline Corte $^{\mathrm{I}}$ & $<25$ & $<100$ & $<29$ & $<125$ & $<35$ & $<3$ & $<4$ & $<4$ & $<5$ & $<4$ & $>90$ \\
\hline Corte $^{\mathrm{II}}$ & & $<109$ & & & $<36$ & $<4$ & & & & & $>81$ \\
\hline GR04 & $22^{\mathrm{a}}$ & $95^{\mathrm{a}}$ & $16^{\mathrm{a}}$ & $111^{\mathrm{a}}$ & $32^{\mathrm{a}, \mathrm{b}}$ & $4^{b}$ & 7 & $1^{\mathrm{a}}$ & 7 & 1 & 80 \\
\hline GR05 & 26 & $80^{\mathrm{a}, \mathrm{b}}$ & $27^{\mathrm{a}}$ & $107^{a}$ & $30^{\mathrm{a}, \mathrm{b}}$ & $3^{\mathrm{b}}$ & $2^{\mathrm{a}}$ & 7 & 6 & 1 & $100^{\mathrm{a}}$ \\
\hline GR06 & $19^{\mathrm{a}}$ & $37^{\mathrm{a}, \mathrm{b}}$ & $11^{\mathrm{a}}$ & $48^{\mathrm{a}}$ & 36 & $2^{\mathrm{a}}$ & 6 & 7 & 5 & $7^{\mathrm{a}}$ & $132^{\mathrm{a}, \mathrm{b}}$ \\
\hline GR10 & 26 & $53^{\mathrm{a}, \mathrm{b}}$ & $20^{\mathrm{a}}$ & $73^{\mathrm{a}}$ & $25^{\mathrm{a}, \mathrm{b}}$ & 3 & $2^{\mathrm{a}}$ & 7 & $4^{a}$ & $4^{\mathrm{a}}$ & 88 \\
\hline GR18 & $21^{\mathrm{a}}$ & $95^{\mathrm{a}, \mathrm{b}}$ & $21^{a}$ & $116^{\mathrm{a}}$ & $33^{\mathrm{a}, \mathrm{b}}$ & 3 & 4 & $4^{\mathrm{a}}$ & 5 & $9^{\mathrm{a}}$ & 88 \\
\hline GR19 & $21^{\mathrm{a}}$ & $90^{\mathrm{a}, \mathrm{b}}$ & $27^{\mathrm{a}}$ & $117^{\mathrm{a}}$ & $25^{\mathrm{a}, \mathrm{b}}$ & $1^{\mathrm{a}}$ & $3^{\mathrm{a}}$ & $2^{\mathrm{a}}$ & $4^{a}$ & $7^{\mathrm{a}}$ & $94^{\mathrm{a}}$ \\
\hline GR22 & $24^{\mathrm{a}}$ & $71^{\mathrm{a}, \mathrm{b}}$ & $28^{\mathrm{a}}$ & $99^{\mathrm{a}}$ & 37 & $2^{\mathrm{a}}$ & 4 & $3^{\mathrm{a}}$ & 5 & 0 & 79 \\
\hline GR24 & 26 & $100^{\mathrm{a}}$ & $24^{a}$ & $124^{\mathrm{a}}$ & $34^{a}$ & 3 & 4 & 7 & 6 & 0 & $91^{\mathrm{a}}$ \\
\hline GR27 & 25 & $23^{\mathrm{a}, \mathrm{b}}$ & $8^{\mathrm{a}}$ & $31^{\mathrm{a}}$ & $25^{\mathrm{a}}$ & 4 & $3^{a}$ & $3^{\mathrm{a}}$ & 5 & 0 & $152^{\mathrm{a}, \mathrm{b}}$ \\
\hline GR30 & $24^{\mathrm{a}}$ & $82^{\mathrm{a}, \mathrm{b}}$ & $22^{\mathrm{a}}$ & $104^{\mathrm{a}}$ & $30^{\mathrm{a}, \mathrm{b}}$ & $3^{b}$ & $2^{\mathrm{a}}$ & $2^{\mathrm{a}}$ & $4^{a}$ & $7^{\mathrm{a}}$ & $110^{\mathrm{a}}$ \\
\hline GR32 & $13^{\mathrm{a}}$ & $20^{\mathrm{a}, \mathrm{b}}$ & $2^{\mathrm{a}}$ & $22^{\mathrm{a}}$ & $33^{\mathrm{a}, \mathrm{b}}$ & $2^{a, b}$ & $3^{\mathrm{a}}$ & $1^{\mathrm{a}}$ & 5 & 2 & $113^{\mathrm{a}, \mathrm{b}}$ \\
\hline GR41 & 26 & $91^{\mathrm{a}}$ & 33 & $124^{\mathrm{a}}$ & 36 & 3 & 6 & 7 & 6 & 1 & 71 \\
\hline GR43 & 25 & $84^{a}$ & $23^{\mathrm{a}}$ & $107^{\mathrm{a}}$ & $32^{\mathrm{a}, \mathrm{b}}$ & 3 & 4 & $1^{\mathrm{a}}$ & $4^{\mathrm{a}}$ & 1 & $71^{\mathrm{b}}$ \\
\hline
\end{tabular}

Nota. ALF- Alfabeto; LEIT- provas de leitura; ESC- provas de escrita; LE- escore composto de leitura e escrita; FON- consciência fonológica; MTF- memória de trabalho fonológica; MID- memória indireta de dígitos; COP- cópia de formas; MV- memória de trabalho visual; ME- memória de trabalho visuoespacial; RAN- nomeação automática rápida.

$0,001)$ e GR $(p<0,002)$ Porém, somente o GC mostrou melhoras significativas na CFO $(p<0,001)$, particularmente nas habilidades de ataque e rima $(p<0,003)$, uma vez que na segmentação silábica não houve melhora significativa em nenhum grupo. Em contraste, somente o GR mostrou melhora significativa na MTFII.

A Tabela 2 permite uma análise individual do progresso de cada escolar ao longo de um ano. Os escolares do grupo de risco (GR) receberam intervenções pedagógicas coletivas realizadas pelo próprio professor em sala de aula, correspondentes à primeira camada do RTI (intervenção primária). Essa intervenção primária corresponde a estratégias pedagógicas baseadas em evidência, tais como prática do alfabeto, ênfase nas relações letra-som, leitura em voz alta de palavras e frases, e outras tarefas da avaliação diagnóstica descrita nas páginas 9 e 10 da seção Material. Os escolares que após a intervenção primária ainda con- tinuaram a apresentar dificuldades, conforme avaliação de rotina da equipe pedagógica, receberam intervenção psicopedagógica secundária em sessões separadas com 3 a 4 alunos, com ênfase em atividades de leitura e escrita focadas nas principais fraquezas apresentadas.

Apenas um escolar do GR (GR41) não apresentou prejuízos em nenhuma das provas do TDCL e respondeu bem às primeiras intervenções psicopedagógicas (primeira camada ou intervenção primária do RTI) saindo do grupo de risco no ano seguinte, de acordo com a $\mathrm{AD}^{\mathrm{II}}$ e TDCL ${ }^{\mathrm{II}}$. Outro escolar (GR24), cujos prejuízos de leitura no TDCL ${ }^{\mathrm{I}}$ eram explicáveis somente por déficits fonológicos, também saiu do grupo de risco, após a segunda camada do RTI (intervenção secundária), de acordo com a $\mathrm{AD}^{\mathrm{II}}$.

Os escolares GR4 e GR43 também tiveram um desempenho acima do ponto de corte nas tarefas de leitura do TDCL ${ }^{\mathrm{II}}$, certamente devido à intensa intervenção psicope- 
Andrade, O. V. C. A., Andrade, P. E. \& Capellini, S. A. (2014). Caracterização do Perfil Cognitivo-Linguístico de Escolares com Dificuldades de Leitura e Escrita.

dagógica (o escolar GR43 também se submeteu a intensa intervenção fonoaudiológica), apesar de continuarem com prejuízos na consciência fonológica no TDCL ${ }^{\mathrm{II}}$. Como estes dois escolares ainda apresentavam certa defasagem na $\mathrm{AD}^{\mathrm{II}}$ e déficits fonológicos no TDCL ${ }^{\mathrm{II}}$, continuaram a ser considerados de risco pela equipe pedagógica no início do $3^{\circ}$ ano. Assim, o percentual de escolares com risco para TL ainda ficou em $24,4 \%$ no início do $3^{\circ}$ ano.

\section{Discussão}

Consistentemente com as correlações moderadas a altas obtidas entre essas habilidades fonológicas (particularmente as de CFO e RAN) e as de leitura e escrita, o grupo GR apresentou desempenho inferior ao GC nas habilidades de leitura e escrita e no processamento fonológico tanto no início do estudo (TDCL ${ }^{\mathrm{I}}$ ) quanto um ano depois (TDCL ${ }^{\mathrm{II}}$ ). Estes dados são consistentes com a noção de que o TL é causado fundamentalmente por prejuízos no processamento fonológico (Ramus et al., 2003; Vellutino, Fletcher, Snowling, \& Scanlon, 2004). Entretanto, a análise individual, utilizando um critério de desvio para cada tarefa do TDCL revela que os déficits atencional e de nomeação podem ser causas independentes de TL.

Critérios mais liberais, como o percentil 25 (25\% dos escores mais baixos, aproximadamente $0,68 \mathrm{DP}$ ) ou 30 (aproximadamente 0,5 DP), ou mais conservadores, como 1,5 DP abaixo da média, são usados para determinar o baixo rendimento (Catts et al., 2001; Fuchs \& Fuchs, 2006). Quanto mais liberal ou mais conservador o critério, maior ou menor será o número de escolares de risco detectados (Fletcher \& Vaughn, 2009; Fuchs \& Fuchs, 2006). Neste estudo foi utilizado um critério mais conservador (escores mais de 1,65 DP abaixo da média de cada grupo controle específico de tarefa, GCE) e mais rigoroso na definição do escolar de risco para TL.

O déficit na consciência fonológica foi o sintoma mais comum, presente em aproximadamente $75 \%$ dos escolares de risco identificados em ambos os períodos (início do $2^{\circ}$ e $3^{\circ}$ anos). Por outro lado o teste $t$ não encontrou efeito de grupo para tarefas relacionadas ao processamento auditivo como a memória indireta de dígitos, a repetição de pseudopalavras e a discriminação auditiva, nem para tarefas envolvendo o processamento visual como a cópia de formas (COP) e a memória de trabalho visual (MVE). A exceção foi um efeito de grupo encontrado para a memória de trabalho visual (MV).

Esses resultados são amplamente consistentes com a visão de que os transtornos de leitura são eminentemente decorrentes de prejuízos na consciência fonológica (Shaywitz \& Shaywitz, 2008; Vellutino et al., 2004). Entretanto, uma análise individual do GR proporcionada pela Tabela 2 , revela um quadro mais completo e um pouco distinto do que a análise de grupo.

Primeiro, apesar do teste $t$ não revelar efeito de grupo para a COP, a análise individual (Tabela 2) revela que 8 escolares, correspondente a $61,5 \%$ do GR, apresentaram prejuízos na COP e 7 deles também apresentaram prejuízos na consciência fonológica em ambos o $\mathrm{TDCL}^{\mathrm{I}}$ e $\mathrm{TDCL}^{\mathrm{II}}$. Isto torna a COP a segunda tarefa com maior sensitividade aos escolares de risco, e, portanto, uma excelente ferramenta não baseada na linguagem escrita de identificação precoce de escolares de risco, que não deve ser desprezada numa abordagem RTI.

Em segundo lugar, constatamos que 5 escolares do GR apresentavam sinais de déficits atencionais e/ou hiperatividade, de acordo com a equipe pedagógica e com a escala de Benczik (Benczik, 2000). A hipótese de TDA/H nesses escolares recebeu suporte adicional do fato de que todos tiveram escores abaixo do ponto de corte na memória de trabalho visuoespacial (MVE), uma evidência típica de déficits atencionais (Castellanos et al., 2006). Nenhum desses escolares com sinais de TDA/H saiu do GR um ano após as intervenções, na $\mathrm{AD}^{\mathrm{II}}$ e no TDCL ${ }^{\mathrm{II}}$, diferentemente dos escolares do GR sem sinais de TDA/H dos quais 4 tiveram desempenho acima do ponto de corte em leitura no TDCL ${ }^{\mathrm{II}}$, e dois deles saíram completamente do risco para TL de acordo com ambos TDCL ${ }^{\mathrm{II}}$ e $\mathrm{AD}^{\mathrm{II}}$.

Estes resultados acima sugerem que a comorbidade dislexia-TDA/H acarreta prejuízos muito mais severos na aquisição de uma língua escrita constituída como sistema alfabético. Particularmente nos subtipos desatento e combinado a comorbidade dislexia-TDA/H associa prejuízos fonológicos e atencionais, o que chama a atenção para a necessidade de se desenvolver estratégias e contextos psicopedagógicos diferenciais para estes casos que, devido à associação da desatenção e/ou hiperatividade, requerem manejo especial nas sessões de intervenção. Dos 5 escolares com sinais de TDA/H um escolar (GR6) representa um caso típico de déficits eminentemente atencionais associados a severos prejuízos de RAN (o segundo menor desempenho em ambas as medições do TDCL), pois teve um desempenho normal na CFO em ambas as medições do TDCL, corroborando, portanto, a literatura recente (Shanahan et al., 2006; Willcutt et al., 2010; Willcutt et al., 2007).

Também encontramos um caso específico de risco para TL decorrente exclusivamente de severos prejuízos na RAN. O escolar GR27 obteve o menor desempenho em RAN de todo os escolares de risco em ambos os TDCL ${ }^{1} \mathrm{e}$ TDCL ${ }^{\text {II }}$, apesar de MTF normal e se destacar nas habilidades de processamento visual e ter um QI acima da média. Embora também apresentasse prejuízo de CFO no TDCL ${ }^{\mathrm{I}}$, um ano após as intervenções, quando foi aplicada a TDCL ${ }^{\mathrm{II}}$, este escolar apresentou prejuízos somente na RAN ${ }^{\mathrm{II}}$ bem como severas dificuldades na leitura, tanto no TDCL ${ }^{\mathrm{II}}$ quanto na $\mathrm{AD}^{\mathrm{II}}$. Este caso corrobora, portanto, a hipótese do duplo-déficit (Wolf \& Bowers, 1999) segundo a qual os prejuízos de CFO e RAN são causas independentes subjacentes ao TL.

Nossos achados revelam que o risco para TL pode ter diferentes causas, tais como o prejuízo na consciência fonológica para 75\% dos escolares (o qual ocorreu associado a déficits atencionais em quase metade desses escolares), o prejuízo exclusivo na nomeação rápida (Wolf \& Bowers, 
1999) e o prejuízo atencional (associado ao prejuízo na nomeação rápida; Shanahan et al., 2006; Willcutt et al., 2010). Esses resultados são plenamente consistentes com a literatura recente sobre a sintomatologia subjacente ao TL, a qual suporta o modelo de múltiplos déficits (várias causas independentes subjacentes ao transtorno de leitura), embora o prejuízo na consciência fonológica responda pela variância na leitura mais do que outras disfunções cognitivas (Willcutt et al., 2010).

Por outro lado, os resultados não suportam a noção de que não há necessidade de se realizar testes que informem sobre o perfil cognitivo-linguístico desses escolares (Fletcher \& Lyon, 2008). Em vez disso, sugerem que o perfil individual de déficits é fundamental para contemplar as necessidades individuais na intervenção (Reynolds \& Shaywitz, 2009).

A comparação intragrupo nos dois momentos de medição do TDCL mostrou que após um ano o grupo GR teve melhoras significativas na leitura (LEIT), memória de trabalho verbal (MTF) e nomeação rápida (RAN), mas não na consciência fonológica (CFO). Uma possível explicação para tal achado é que as estratégias psicopedagógicas utilizadas nas intervenções não utilizaram atividades específicas para o desenvolvimento da CFO, mas se basearam primordialmente na ênfase das relações letra-som.

Finalmente, nesta investigação todos escolares com prejuízos atencionais continuaram sendo de risco mesmo após um ano de intervenção. Este padrão é consistente com estudos mostrando que a comorbidade com transtorno de leitura pode ser indicador de um grupo de TDA/H com déficits cognitivos mais severos (Willcutt et al., 2010; Willcutt et al., 2001; Willcutt et al., 2007).

\section{Conclusão}

O prejuízo na consciência fonológica responde pela variância na leitura mais do que outras disfunções cognitivas. Porém, o risco para transtornos de leitura possui diferentes causas independentes, além de prejuízos na consciência fonológica, tais como prejuízos na nomeação rápida e desatenção, os quais estão associados a prejuízos na memória de trabalho fonológica e nomeação rápida, consistentemente com o modelo de múltiplos déficits explicativo da dislexia. Concluímos a favor de um modelo ampliado de resposta à intervenção, isto é, que ao invés de basear-se apenas no seu desempenho escolar / ou acadêmico também identifique o perfil cognitivo-linguístico de cada escolar. Um modelo ampliado que contemple a identificação precoce das forças e fraquezas cognitivas de cada escolar de risco para transtorno de leitura permitirá o desenvolvimento de programas de intervenção precoce mais eficazes, tanto na orientação de estratégias psicopedagógicas mais adequadas para o desenvolvimento de habilidades específicas relevantes para a aquisição da linguagem escrita (e.g., habilidades de processamento fonológico), como na orientação do manejo adequado do ambiente psicopedagógico tão fundamental no caso de déficits atencionais.

\section{Referências}

Almeida, P. M., \& Wickerhauser, H. (1991). O critério ABA/ABIPEME: Em busca de uma atualização. São Paulo, SP: LPM.

Alves, L. M., Mousinho, R., \& Capellini, S. (2011). Dislexia. Novos temas, novas perspectivas. Rio de Janeiro, RJ: Wak.

Alves, L. M., \& Ribeiro, M. M. (2011). Desempenho em memória de trabalho em escolares com e sem queixas de alterações de aprendizagem. Tecer, 4, 54-65.

Alves, L. M., Siqueira, C. M., Lodi, D. F., \& Araújo, M. C. M. F. (2011). Introdução à dislexia do desenvolvimento. In L. M. Alves, R. Mousinho, \& S. A. Capellini (Eds.), Dislexia. Novos temas, novas perspectivas (pp. 21-40). Rio de Janeiro, RJ: Wak.

American Psychiatric Association. (2002). DSM-IV-TR: Manual diagnóstico e estatístico de transtornos mentais (4. ed. rev.). Porto Alegre, RS: Artmed.

Andrade, O. V. C. A., Prado, P. S. T., \& Capellini, S. A. (2011). Desenvolvimento de ferramentas pedagógicas para identificação de escolares de risco para a dislexia. Revista Psicopedagia, 28(85), 14-28.

Benczik, E. B. P. (2000). Manual da escala de transtorno de déficit de atenção/ hiperatividade: Versão para professores. São Paulo, SP: Casa do Psicólogo.

Bicalho, L. G. R., \& Alves, L. M. (2010). A nomeação seriada rápida em escolares com e sem queixas de problemas de aprendizagem em escola pública e particular. Revista CEFAC, 12, 608-616.

Capellini, S. A., Sampaio, M. N., Matsuzawa, M. T., Oliveira, A. M., Fadini, C. C., \& Martins, M. A. (2009). Protocolo de identificação precoce dos problemas de leitura: Estudo preliminar com escolares de $1^{\circ}$ ano escolar. Revista Psicopedagogia, 26(80), 367-75.

Capellini, S. A., \& Smythe, I. (2008). Protocolo de avaliação de habilidades cognitivo-linguísticas: Livro do profissional e do professor. Marília, SP: Fundação para o Desenvolvimento do Ensino, Pesquisa e Extensão.

Castellanos, F. X., Sonuga-Barke, E. J., Milham, M. P., \& Tannock, R. (2006). Characterizing cognition in ADHD: Beyond executive dysfunction. Trends in Cogntive Sciences, 10(3), 117-23.

Catts, H. W., Fey, M. E., Zhang, X., \& Tomblin, J. B. (2001). Estimating the risk of future reading difficulties in kindergarten children: A research-based model and its clinical implementation. Language, Speech, and Hearing Services in Schools, 32, 38-50.

Ciasca, S. M. (2004). Distúrbios de aprendizagem: Proposta de avaliação interdisciplinar. São Paulo, SP: Casa do Psicólogo.

Demo, P. (2007). Escola pública e escola particular: Semelhanças de dois imbróglios educacionais. Ensaio: Avaliação e Políticas Públicas em Educação, 15(55), 181-206.

Fletcher, J. M., \& Lyon. G. R. (2008). Dyslexia: Why precise definitions are important and how we have achieved them. Perspectives on Language and Literacy, 34, 27-31.

Fletcher, J., \& Vaughn, S. (2009). Response to intervention: Preventing and remediating academic difficulties. Child Development Perspectives, 3, 30-37.

Foster, G. (1986). Financial statement analysis ( $2^{\text {nd }}$ ed.). Englewood Cliffs, NJ: Prentice-Hall.

Fuchs, D., \& Fuchs, L. S. (2006). Introduction to response to intervention: What, why, and how valid is it? Reading Research Quarterly, 41, 93-99. 
Andrade, O. V. C. A., Andrade, P. E. \& Capellini, S. A. (2014). Caracterização do Perfil Cognitivo-Linguístico de Escolares com Dificuldades de Leitura e Escrita.

Luckesi, C. C. (2005). Avaliação da aprendizagem na escola: Reelaborando conceitos e criando a prática (2. ed). Salvador, BA: Malabares Comunicações e Eventos.

Martinussen, R., \& Tannock, R. (2006). Working memory impairments in children with attention-deficit hyperactivity disorder with and without comorbid language learning disorders. Journal of Clinical and Experimental Neuropsycology, 28(7), 10073-1094.

Ramus, F., Rosen, S., Dakin, S. C., Day, B. L., Castellote, J. M., White, S., \& Frith, U. (2003). Theories of developmental dyslexia: Insights from a multiple case study of dyslexic adults. Brain. 126, 841-65.

Refundini, D. C., Martins M. A., \& Capellini S. A. (2010). Treinamento da correspondência grafema-fonema em escolares de risco para a dislexia. Revista Psicopedagogia, 27(83), 191-201.

Reynolds, C. R., \& Shaywitz, S. E. (2009). Response to intervention: Ready or not? Or, from wait-to-fail to watch-them-fail. School Pschology Quaterly, 24, 130-145.

Shanahan, M. A., Pennington, B. F., Yerys, B. E., Scott, A., Boada, R., Willcutt, E. G., ...DeFries, J. C. (2006). Processing speed deficits in attention deficit/hyperactivity disorder and reading disability. Journal of Abnormal Child Psychology, 34, 585-602.

Shaywitz, S. E., Morris, R., \& Shaywitz, B. A. (2008). The education of dyslexic children from childhood to young adulthood. Annual Review of Psychology, 59, 451-475.

Shaywitz, S. E., \& Shaywitz, B. A. (2008). Paying attention to reading: The neurobiology of reading and dyslexia. Development and Psychopathology, 20, 1329-1349.

Siqueira, C. M., Gurgel-Giannetti, J., Araújo, M. C. F. M., Lodi, D. F., Alves, J. F. M., Avelar, K., \& Alves, L. M. (2011) Transtorno de deficit de atenção/hiperatividade e transtornos de aprendizagem. In L. M. Alves, R. Mousinho, \& S. A. Capellini (Eds.), Dislexia: Novos temas, novas perspectivas (pp. 283-301). Rio de Janeiro, RJ: Wak.

Vellutino, F. R., Fletcher, J. M., Snowling, M. J., \& Scanlon, D. M. (2004). Specific reading disability (dyslexia): What have we learned in the past four decades? Journal of Child Psychiatry and Psychology and Allied Disciplines, 45, 2-40.

Willcutt, E. G., Betjemann, R. S., McGrath, L. M., Chhabildas, N. A., Olson, R. K., DeFries, J. C., \& Pennington, B. (2010) Etiology and neuropsychology of comorbidity between RD and ADHD: The case for multiple-deficit models. Cortex, 46(10), 1345-1361.

Willcutt, E. G., Pennington, B. F., Boada, R., Ogline, J. S. Tunick, R. A., Chhabildas, N. A., \& Olson, R. K. (2001). A comparison of the cognitive deficits in reading disability and attention-deficit/hyperactivity disorder. Journal of Abnormal Psychology. 110, 157-172.

Willcutt, E. G., Pennington, B. F., Olson, R. K., \& DeFries, J. C. (2007). Understanding comorbidity: A twin study of reading disability and attention-deficit/hyperactivity disorder. American Journal of Medical Genetics Part B: Neuropsychiatric Genetics, 144B(6), 709-714.

Wolf, M., \& Bowers, P. G. (1999). The double-deficit hypothesis for the developmental dyslexias. Journal of Educational Psychology, 91(3), 415-438. 